\title{
Orthodontic diagnosis based upon alveolar bone clinical morphology
}

\begin{abstract}
The 120-year old classification developed by Edward Angle has largely been sustained to the present day as the main language of malocclusion. It serves as the basis for the orthodontic treatment goal of attaining a specific molar relation arbitrarily defined as "ideal" with an accompanying prescribed facial profile. This classification is not biologically based and was founded on social observation of Caucasians thereby not accounting for natural variations across ethnic groups and race. The authors believe it misdirects treatment and propose new biologically based diagnostic terms centered on the pretreatment clinical morphology of the alveolar bone. Logically, treatment is based on improving the alveolar bone morphology by maintaining a stable occlusion irrespective of molar class with a novel patented orthodontic system.
\end{abstract}

Keywords: orthodontics, alveolar bone morphology, maxillary and mandibular hypoplasia, maxillary and mandibular hyperplasia, orthoeruption, orthodontosis, orthodontitis
Volume 8 Issue 8 - 2017

\author{
Anthony DViazis,' Evangelos Viazis, ${ }^{2}$ Tom C \\ Pagonis $^{3}$ \\ 'Orthodontist, Private Practice, Dallas, Texas, USA \\ 2Private Practice, Athens, Greece \\ ${ }^{3}$ Assistant Professor (former), Harvard School of Dental \\ Medicine, Boston, MA, USA
}

Correspondence: Tom C Pagonis, Assistant Clinical Professor (Former), Harvard School of Dental Medicine, Boston, MA, USA, Email dental.research@comcast.net

Received: December 13, 2017| Published: December 27, 2017

\section{Introduction}

Growing scientific evidence which directly refutes the basis for conventional diagnostic classification of malocclusion along with dramatic improvements in the design of orthodontic brackets and implementation of new orthodontic techniques clearly justify the need for biologically driven orthodontic diagnoses. Angle's 120year old classification of malocclusion. ${ }^{1}$ has remarkably endured and continues to be utilized as the main language of malocclusion among orthodontic specialists in spite of its lack of verifiable scientific validity. ${ }^{2-6}$ In effect, an arbitrary or subjective concept of ideal occlusion based upon Angle's classification of malocclusion serves as the basis for orthodontic treatment planning for a large majority of orthodontic providers around the world. Yet there is no evidence to suggest that this arbitrary "ideal" occlusion provides significant health benefits or that it significantly improves oral function. Furthermore, Angle's classification is not based on a verifiable biologically based constant but relies on dental intercuspation or static occlusion which takes place between 15 to 30 minutes per day. ${ }^{7}$ This only represents a static glimpse of a 24-hour cycle of dynamic occlusal function. Cusps of teeth do not possess an innate or pre-programmed function to articulate in a specific way or position. Furthermore, cusps do not know Angle's classifications and adapt to a functional occlusal with maximum intercuspation irrespective of the anterior-posterior position of the mesio- buccal cusp of the maxillary first molar relative to the mandibular first molar. Angle's classification of malocclusion can more appropriately be referred to as social observations rather than pathologic diseases and changes to stable occlusions during orthodontic treatment to achieve a morphologic occlusion that conforms to a subjective or arbitrary" ideal" remains questionable. We therefore propose orthodontic diagnoses of malpositioned teeth based upon the pretreatment clinical morphology of the alveolar bone and accompanying orientation of tooth roots. The alveolar bone morphology is a biologically based constant and a logical element to utilize in the diagnostic process.

\section{Materials and methods}

\section{Etiology and prevalence of malocclusion}

In 1771, John Hunter, a British anatomist was one of the first to explain normal occlusion and described the growth of jaws. ${ }^{8}$ Addressing the complex nature of etiology requires an appreciation and even an agreement on developing criteria for a normal occlusion. Contemporary attempts to classify normal occlusion have been forwarded by Andrews, ${ }^{9}$ Roth, ${ }^{10}$ along with Ash \& Ramjford. ${ }^{11}$ Rinchuse et al. ${ }^{12}$ introduced the functional concept of occlusion directly challenging "experience-based" rather than evidence-based concepts first proposed by Angle. Several classification methods have been proposed in an attempt to categorize the etiology of malocclusion. This includes Moyers' classification. ${ }^{13}$ which identifies heredity, trauma, physical agents, habits, diseases, malnutrition and developmental defects of unknown origin. In addition, Graber's classification. ${ }^{14}$ divided etiologic factors into two group's namely general factors and local factors. General factors include heredity, congenital defects, and environment, predisposing metabolic diseases, dietary problems, abnormal habits, posture and trauma. Local factors include anomalies of tooth number (i.e. supernumerary or missing teeth), anomalies of tooth size and shape, abnormal labial frenum, along with delayed or abnormal eruption of permanent teeth. Finally, Ackerman and Proffit's classification. ${ }^{15}$ proposed three main groups; namely

i. Specific causes

ii. Genetic influences

iii. Environmental influences. For specific causes, Ackerman and Profitt proposed disturbances in embryologic development, skeletal growth disturbances, muscle dysfunction, acromegaly and disturbances in dental development. Environmental influences include masticatory function, oral habits and respiratory pattern. These classifications exhibit the complexity and varying 
interdependence of both hereditary and environmental factors when discussing the etiology of malocclusion. The prevalence of malocclusion particularly among children exhibits a broad range from $39 \%$ to $93 \% .{ }^{16-19}$ This wide range reflects variations in registration of malocclusions, identifying varying degrees of malocclusions (i.e. "mild" to "severe"), age and variations among ethnic groups.

\section{Proposed orthodontic diagnoses}

\section{Maxillary or mandibular alveolar hypoplasia}

This clinical presentation typically exhibits the appearance of lingually malpositioned roots of teeth. This is accompanied by clinical deficiency or hypoplasia of the alveolar bone in the labial/buccal area of the affected teeth. This gives the appearance of crowding when observing the clinical crowns - hence the use of old quantifying diagnostic terms of "slight", "moderate" or "severely" crowded teeth. The level of crowding severity is no longer relevant as nearly all non-skeletal cases can be treated non extraction. The specific loss of localized normal boney architecture and associated localized soft tissue inflammatory changes caused by malpositioned roots has been termed orthodontosis and orthodontitis, respectively. ${ }^{6}$

\section{Maxillary or mandibular alveolar hyperplasia}

While the etiology of tooth or dental spacing is multifactorial and can manifest via microdontia or the size of teeth along with physiologic habits such as thumb sucking and tongue thrust alveolar size is the primary factor that determines orientation of teeth. Current thought suggests that dental spacing from tongue thrust habits may be a consequence of rather than the cause of an anterior open bite. ${ }^{20}$ The clinical presentation of this diagnosis logically is spacing of teeth especially of anterior teeth with normal architecture of the alveolar bone and normal intraboney orientation of all tooth roots. Dental spacing between anterior teeth is always seen but often times it is not seen with premolar teeth. One strong possibility for lack of spacing in premolar teeth is the function of the buccinator muscle with its proximity to the alveolar bone and dental arches as discussed in classic studies. ${ }^{21}$ Brackets are therefore often not required for teeth exhibiting normal spacing or are in proximal contact.

\section{Addition of occlusal factors}

The above referenced diagnoses would also include traditional static occlusion addendums of cross-bite, anterior open bite with specific measurements of overbite and overjet. The authors believe that recording molar relationship is not necessary particularly for a stable occlusion because the goal of orthodontic treatment should not be to change the molar relationship in pursuit of an arbitrary occlusal morphology. What's more important is to create a functional and esthetic result by addressing an appropriate overbite/overjet of 1 to $3 \mathrm{~mm}$ utilizing non-extraction therapy.

\section{Report of cases}

Four adult patients, seen by four different providers presented for orthodontic treatment with new orthodontic diagnostic terms of maxillary and mandibular hypoplasia with localized orthodontosis and orthodontitis (Figures 1 \& 2), and maxillary and mandibular hyperplasia (Figures $3 \& 4$ ) were successfully treated with the patented systems of FASTBRACES ${ }^{\circledR}$ Technologies. It is important to note that the universal orthodontic goal and accompanying treatment should be to successfully treat the biologically based diagnosis of the alveolar bone clinical morphology within a patient's natural stable occlusion and morphologic appearance. Of note is that each of the maxillary and mandibular hypoplasia cases (Figures 1 \& 2) started with an Angle Class I and Class II malocclusion but treatment was successfully directed towards addressing deficiencies of the alveolar bone morphology while achieving an overbite/overjet relation of 1 to $3 \mathrm{~mm}$. In similar fashion each maxillary and mandibular hyperplasia (Figures 3 \& 4) started with an Angle Class I and Class II malocclusion but treatment was successfully directed towards addressing the spacing of teeth while achieving an overbite/overjet relation of 1 to $3 \mathrm{~mm}$. Therefore, the pre and post treatment molar relationship is of no relevance and should not direct treatment. In fact, it would be difficult to determine the molar relationship of these cases when comparing post treatment photos (Figure 5). This is because the universal constant is the alveolar bone clinical morphology with treatment directed towards the alveolar bone deficiencies when present and not the molar relationships. These four cases are successful examples of non- extraction orthodontic treatment with the patented systems of FASTBRACES ${ }^{\circledR}$ Technologies which appropriately address the relevant deficiencies in the alveolar bone clinical morphology while maintaining a 1 to $3 \mathrm{~mm}$ overbit /overjet and not changing the patient's molar relation. The authors believe the systems of FASTBRACES ${ }^{\circledR}$ Technologies induce alveolar bone remodeling by moving the tooth roots towards their natural properly erupted positions from the onset of treatment.
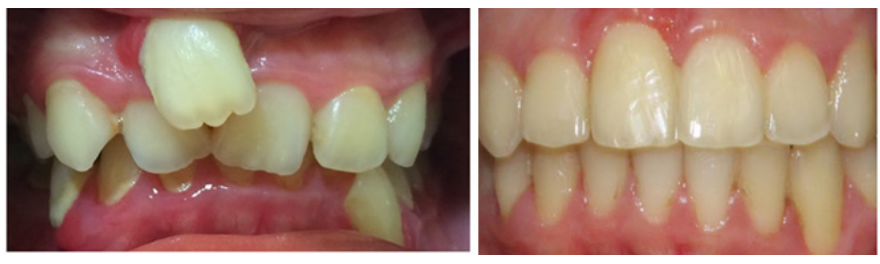

Figure IA before Orthodontic Diagnosis of Maxillary and Mandibular Hypoplasia. Figure IB after Natural occlusion achieved with an overbite loverjet relation of I to $3 \mathrm{~mm}$. The Class I molar relation of this case was irrelevant.
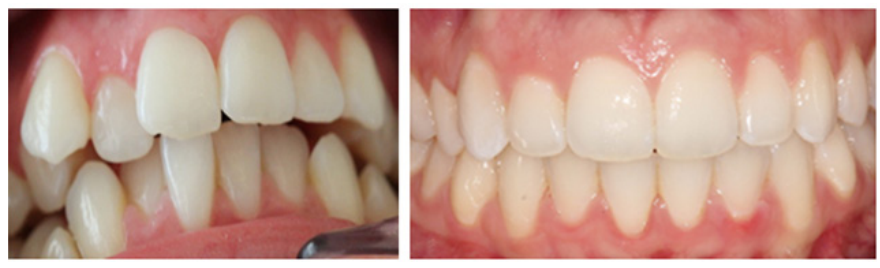

Figure 2A before Orthodontic Diagnosis of Maxillary and Mandibular Hypoplasia. Figure 2B after Natural occlusion achieved with an overbite loverjet relation of I to $3 \mathrm{~mm}$. The Class II molar relation of this case was irrelevant.
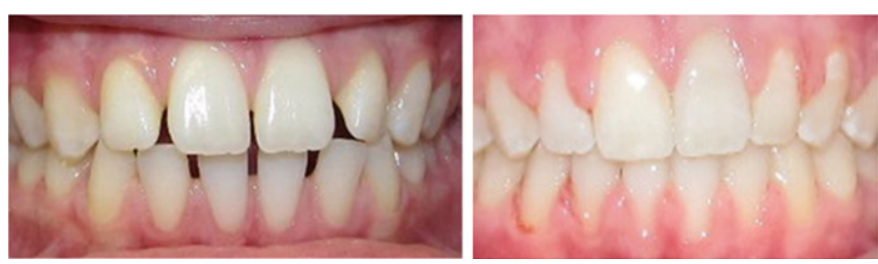

Figure 3A before Orthodontic Diagnosis of Maxillary and Mandibular Hyperplasia. Figure 3B after Natural occlusion achieved with an overbite loverjet relation of $\mathrm{I}$ to $3 \mathrm{~mm}$. The Class I molar relation of this case was irrelevant. 


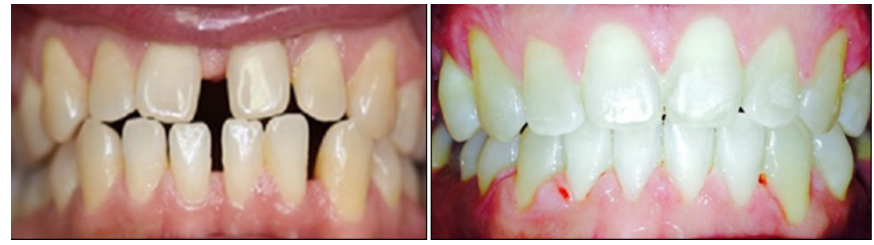

Figure 4A before Orthodontic Diagnosis of Maxillary and Mandibular Hyperplasia. Figure 4B after Natural occlusion achieved with an overbite loverjet relation of I to $3 \mathrm{~mm}$. The Class III molar relation of this case was irrelevant.

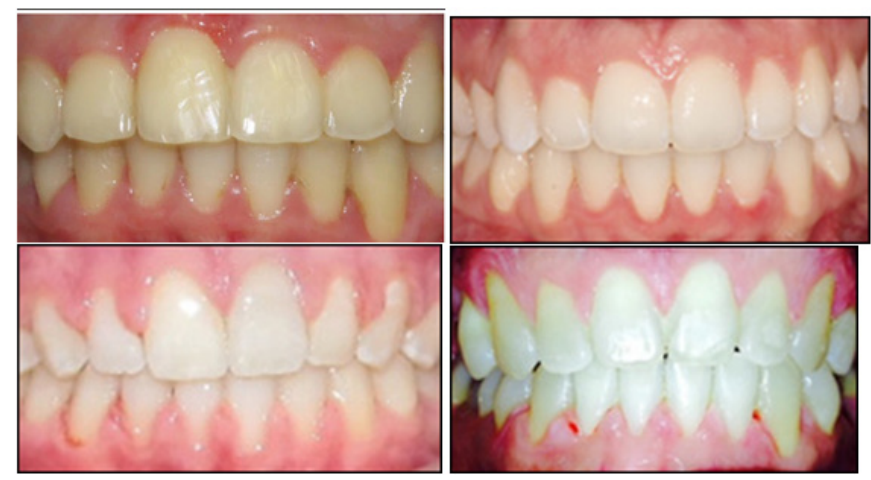

Figure 5 The post treatment photographs of the cases presented in Figures I- 4 look about the same and demonstrate an overjet / overbite relationship of I to $3 \mathrm{~mm}$, irrespective of their molar relation.

\section{Discussion}

The ultimate goal of orthodontic treatment is based upon the premise of improving function, dental and facial esthetics and maintaining or improving dental health. The authors therefore believe that orthodontic diagnosis should be based upon a biological constant which logically is represented by the pretreatment clinical morphology of the alveolar bone. Unfortunately, this has not been the case. From a historical perspective and dating back as far back as 1829 (Samuel S. Fitch) the clinical presentation of "crooked teeth" is not diagnosed with a biological etiology but primarily classified on the basis of static occlusion. ${ }^{22}$ In 1899 Edward Angle. ${ }^{1}$ shaped orthodontic thinking on occlusion by maintaining that the predictability and consistency of the maxillary first molar eruption pattern was of paramount importance. This represented the fundamental underpinning of Angle's philosophy and one that has been enthusiastically acknowledged as doctrine. While modifications have been made, Angle's classification has remarkably endured for over a century with occlusion of teeth being the sole benchmark of normalcy. In 1907 Angle. ${ }^{23}$ stated that the fundamental scientific treatment goal of orthodontics is "the correction of the malocclusions of the teeth". Edward Angle based his classification of malocclusion on a small localized population sample size which lacked racial diversity. Based upon these limited observations and non-biological etiology, Angle advocated a treatment goal of obtaining an "ideal" occlusion based on a specific molar relation and accompanying straight line facial profile which was arbitrarily based on the statue of the mythical Greek God Apollo. The "ideal" occlusion defined by Angle largely consists of the universal patient attainment of a specific or Angle Class 1 molar relation irrespective of racially different patient profiles. Classification systems of malocclusion can best be described as a grouping of similar appearing clinical cases for the sole purpose of discussion. These classifications are not diseased based, do not represent a system of diagnosis, are not a method for assessing treatment prognosis and certainly not an appropriate way of defining treatment. They are merely social observations with treatment goals largely based on altering or even camouflaging the impact of the classified malocclusion. They are not biologically based and almost exclusively derived from observations and treatment of Caucasian patients. One would therefore even have to question the value of some cephalometric "norms" or "averages".

A clinician should consider that the premise of treating an observed malocclusion towards an arbitrary "ideal" is a misdirection of treatment. This is problematic for a variety of reasons; one being that variations of natural profiles exist along racial lines and treatment protocols broadly intended for a Caucasian patient could cause unnecessary dentoalveolar mutilation through extraction therapy with an unfavorable change in facial profile especially if a non-Caucasian patient was only concerned about his or her esthetic of "crooked teeth". For example, African American patients exhibit a higher prevalence of bimaxillary protrusion, larger teeth and even wider faces ${ }^{24-26}$ which manifests as a pronounced soft tissue protrusion. It is clear that an orthodontic treatment plan based upon strict adherence to attaining occlusion of a specific molar relation and with a specific facial profile is undesirable especially if an African American patient does not want to change his or her profile. Besides facial profile differences between African Americans and Caucasians there are differences among other races including Asians, American Indian or Alaska Native and Native Hawaiian or other Pacific Islander. Researchers have recognized the need to perform additional clinical analyses. ${ }^{27-30}$ to evaluate differences in order to ascertain normative values among race and ethnic groups. The authors of this paper maintain that orthodontic diagnosis and subsequent treatment planning should be based on a biologically based constant which is the pretreatment clinical morphology of the alveolar bone. The problem of using a first molar relation in orthodontic "diagnosis" is further brought into light by directly comparing it to the ADA (American Dental Association) /AAP (American Academy of Periodontology) classifications in periodontal disease. For example, an AAP class/type III periodontal diagnosis in part means probing depths or attachment loss of 4 to $6 \mathrm{~mm}$. This periodontal classification and accompanying clinical findings are universal among all patients irrespective of race or ethnicity. Treatment that follows is universal. An Angle III malocclusion is described as an abnormal anteroposterior dental discrepancy with the mesio-buccal cusp of a maxillary first molar articulating distal to the mandibular buccal groove. For a Japanese person it is more prevalent and can be considered a normal craniofacial finding compared to a Caucasian. ${ }^{31}$ Treatment that follows is therefore not universal. We therefore propose orthodontic diagnostic terms of Maxillary or Mandibular Alveolar Hypoplasia and Maxillary or Mandibular Alveolar Hyperplasia. This creates a diagnostic and treatment philosophy which is based on accepting the patient's natural dentition within their own individual genetic morphologic appearance rather than subjective or arbitrary ideals. The amount of severity of teeth crowding or spacing is irrelevant in almost all non-skeletal cases because most treatments are typically completed non extraction. In the case of Alveolar Hypoplasia (maxillary or mandibular) treatment with the patented systems of FASTBRACES ${ }^{\circledR}$ Technologies initially addresses the characteristic non-inflammatory pattern of the alveolar bone hypoplasia known as orthodontosis which is associated with incomplete eruption and lingually malpositioned roots of teeth. Brackets and wires are placed on all teeth that are lingually displaced and exhibit alveolar bone hypoplasia/ orthodontosis. These innovative systems facilitate what the authors 
believe to be the continuation of natural eruption or Orthoeruption. ${ }^{6}$ by inducing alveolar bone remodeling and development by moving roots toward their final naturally erupted position from the beginning of treatment. In the case of Alveolar Hyperplasia, (maxillary or mandibular) treatment with the patented systems of FASTBRACES ${ }^{\circledR}$ Technologies follows a similar sequence of attaching brackets and wires to the teeth exhibiting clinical spacing and closing of these spaces with elastic power chains. In summary, changing the molar relationship especially in a stable functional occlusion should not be the driving factor in orthodontic treatment. Rather, attention to the pretreatment alveolar clinical morphology should be the driving force of a biologically based orthodontic diagnosis along with the functional goal of 1 to $3 \mathrm{~mm}$ of overjet/overbite. The clinician with the appreciation of a patient follows an esthetic and functional non extraction orthodontic treatment based upon correcting or improving the alveolar bone clinical morphology by moving tooth roots from the onset of treatment thereby maintaining patient specific natural facial morphology. Successful treatment which specifically addresses this philosophy in markedly reduced treatment times has been attained across a variety of clinical presentations with the patented systems of FASTBRACES ${ }^{\circledR}$ Technologies..$^{20,32-36}$

\section{Conclusion}

Proposing orthodontic diagnostic terms based upon the pretreatment clinical morphology of the alveolar bone fulfills several important considerations. This includes utilizing the biologically based constant of alveolar bone rather than reproducing an arbitrary "ideal" occlusion which conforms to a molar relation derived from observation of static occlusion which occurs approximately 15 to 30 minutes per day. This only represents a static glimpse of a 24-hour cycle of dynamic occlusal function. Utilizing clinical morphology of the alveolar bone logically follows into universal orthodontic treatment which accepts a patient's natural morphologic appearance and stable occlusion irrespective of molar relationships towards an improved alveolar morphology by moving malpositioned tooth roots from the onset of treatment thereby inducing alveolar bone remodeling and development.

\section{Acknowledgments}

None.

\section{Conflicts of interest}

None.

\section{References}

1. Angle EH. Classification of malocclusion. Dental Cosmos. 1989;41(3):248-226.

2. Rinchuse D, Rinchuse D. Ambiguities of Angle's classification. The Angle Orthodontist. 1989;59(4):295-298.

3. Gravely JF, Johnson DB. Angle's classification of malocclusion:an assessment of reliability. Br J Orthod. 1974;1(3):79-86.

4. Siegel NA. A matter of class:interpreting sub-division in a malocclusion. Am J Orthod Dentofac Orthop. 2002;122(6):582-586.

5. Kato T, Thie NM, Huynh N, et al. Topical review:sleep bruxism and the role of peripheral sensory influences. J Orofacial Pain. 2003;17(3):191213.

6. Viazis AD, Viazis E, Pagonis TC. The Concept of a New Dental Disease:Orthodontosis and Orthodontitis. J Dental Health Oral Disord Therapy. 2014;1(5):00030.
7. Dyer GS, Harris EF, Vaden JL. Age effects contrasted on orthodontics with adult treatment:Adolescents. Am J Orthod Dentofacial Orthop. 1991;100(6):523-530.

8. Hunter J. The Natural History of the Human Teeth:Explaining Their Structure, Use, Formation, Growth, and Diseases. To which is Added, a Practical Treatise on the Diseases of the Teeth. $3^{\text {rd }}$ edn, USA: J. Johnson; 1778. pp. 246.

9. Andrews LF. The six keys to normal occlusion. Am J Orthod. 1972;62(3):296-309.

10. Roth RH. Functional occlusion for the orthodontist. J Cli Orthod. 1981;15(1):32-51.

11. Ash MM, Ramfjord S. Occlusion. $4^{\text {th }}$ edn. USA: W.B. Saunders; 1996.

12. Rinchuse DJ, Kandasamy S, Sciotec J. A contemporary and evidencebased view of canine protected occlusion. Am J Orthod Dentofacial Orthop. 2007;132(1):90-102.

13. Moyers, Michael L Riolo, et al. Facial types associated with Class II malocclusion. Am J Orthod. 1980;78(5):477-494.

14. Graber TM. The "three M's":Muscles, malformation, and malocclusion. Am J Orthod. 1963;49(6):418-450.

15. Ackerman JL, Profitt WR. The characteristics of malocclusion:a modern approach to classification and diagnosis. Am J Orthod. 1969;56(5):443454.

16. Dimberg L, Lennartsson B, Soderfeldt B, et al. Malocclusions in children at 3 and 7 years of age:a longitudinal study. Eur J Orthod. 2013;35(1):131-137.

17. Thilander B, Pena L, Infante C, et al. Prevalence of malocclusion and orthodontic treatment need in children and adolescents in Bogota, Colombia. An epidemiological study related to different stages of dental development. Eur J Orthod. 2001;23(2):153-167.

18. Thilander B, Myrberg N. The prevalence of malocclusion in Swedish schoolchildren. Scand J Dent Res. 1973;81(1):12-21.

19. Tschill P, Bacon W, Sonko A. Malocclusion in the deciduous dentition of Caucasian children. Eur J Orthod. 1977;19(4):361-367.

20. Viazis AD, Viazis E, Pagonis TC. The Fallacy of Tongue Thrust and Non-Surgical Treatment of a Severe Anterior Open Bite. J Dental Health Oral Disord Therapy. 2016;(4):00120.

21. Fränkel R. Decrowding during eruption under the screening influence of vestibular shields. Am J Orthod. 1974;65(4):372-406.

22. Fitch SS. A system of dental surgery:in three parts. G. \& C. \& H. Carvill, USA. 1829.

23. Angle EH. Treatment of malocclusion of the teeth. Angle's system. greatly enl. and entirely rewritten, with six hundred and forty-one illustrations. $7^{\text {th }}$ edn. Philadelphia, USA: S.S. White Manufacturing Co; 1907. pp. 628.

24. Altemus LA. Frequency of the incidence of malocclusion in American Negro children age twelve to sixteen. Angle Orthod. 1959;29(4):189-200.

25. Fonseca RJ, Klein WD. A cephalometric evaluation of American Negro women. Am J Orthod. 1978;73(2):152-160.

26. Nummikoski P, Prihoda T, Langlais RP, et al. Dental and mandibular arch widths in three ethnic groups in Texas:a radiographic study. Oral Surg Oral Med Oral Pathol. 1988;65(5):609-617.

27. Reilly MT. Integumental profile changes after surgical orthodontic correction of bimaxillary dentoalveolar protrusion in black patients. Am J Orthod Dentofacial. 1989;96(3):242-248.

28. Mora S, Boechat MI, Pietka E, et al. Skeletal age determinations in children of European and African descent:applicability of the Greulich and Pyle standards Pediatr Res. 2001;50(5):624-628. 
29. Soegiharto BM, Cunningham SJ, Moles DR. Skeletal maturation in Indonesian and white children assessed with hand-wrist and cervical vertebrae methods. Am J Orthod Dentofacial Orthop. 2008;134(2):217226.

30. Montasser, Viana G, Evans CA. Racial and sex differences in timing of the cervical vertebrae maturation stages. Am J Orthod Dentofacial Orthop. 2017;151(4):744-749.

31. Ishii N, Toshio Deguch, Nigel P Hun (2002) Craniofacial differences between Japanese and British Caucasian females with a skeletal Class III malocclusion. European Journal of Orthodontics. 2002;24:493-499.

32. Viazis AD, Viazis E, Pagonis TC. Non-Surgical Orthodontic Treatment of an Orthognathic Surgical Case. J Dent Health Oral Disord Ther. 2016;4(5):00128
33. Viazis AD, Viazis E, Pagonis TC. Non-surgical Orthodontic Adult Molar Crossbite Correction and Sleep Apnea. J Dent Health Oral Disord Ther. 2016;5(5):00168.

34. Viazis AD, Thaveeprungsiporn M, Pagonis TC. Alveolar Bone Remodeling and Development after Immediate Orthodontic Root Movement. J Dent Health Oral Disord Ther. 2017;6(2):00195.

35. Viazis AD, Viazis E, Pagonis TC. Alveolar Bone Growth with Orthoeruption. J Dent Health Oral Disord Ther. 2017;7(5):00258.

36. Viazis AD, Viazis E, Pagonis TC. The Biology of Orthodontic Treatment Time:Days Versus Years. J Dent Health Oral Disord Ther. 2017;8(1):00268. 